\title{
Factores endocrinos involucrados en la divergencia y la dominancia folicular en bovinos
}

Endocrine factors involved in divergence and dominant follicle in cattle

Fatores endócrinos involucrados na divergência e dominância folicular em bovinos

\author{
Ingris Yohana Hernández Martínez, MVZ, MSc. ${ }_{1}$ \\ Jenny Johanna Pacheco López, Ing. Esp. \\ Ana Paula Castro Santos, MV. MSc \\ José Nelio de Souza Sales, MV, MSc, $\mathrm{PhD}_{3,4}$
}

Recibido: 19 de julio de 2020

Aprobado: 26 de agosto de 2020

Publicado: 15 de noviembre de 2020

Cómo citar este artículo:

Hernández-Martínez IY, Pacheco-López J, Castro Santos, AP, de Souza-Sales JN. Factores endocrinos involucrados en la divergencia y la dominancia folicular en bovinos.

Spei Domus. 2020;16(2): 1-16.

doi: https://doi.org/10.16925/2382-4247.2020.02.01

Artículo de revisión. https://doi.org/10.16925/2382-4247.2020.02.01

1 Universidad Popular del Cesar, Seccional Aguachica, Colombia.

Correo electrónico: iyhernandez@unicesar.edu.co

Univeridade Federal de Lavras, Lavras, Brasil.

3 Universidade Federal de Juiz de Fora, São Pedro. Brasil. 


\section{Resumen}

El propósito de este artículo es proporcionar al lector conocimientos sobre la relación de crecimiento entre el futuro folículo dominante FD, el folículo subordinado (FS) y los factores intraovarianos que inciden en la divergencia folicular en los bovinos.

Desarrollo. Esta revisión inicia presentando la importancia de la divergencia folicular, momento en el cual se observa una diferencia significativa en la tasa de crecimiento entre los dos primeros folículos. Seguidamente se abarcan los aspectos que ejercen influencia en dicha divergencia: presencia del cuerpo lúteo (CL), su relación con el folículo dominante (FD) y las concentraciones plasmáticas de progesterona (P4), además de los factores necesario realizar mas investigaciones para continuar descubriendo nuevos factores que interactuan en este proceso.

Palabras claves: cuerpo lúteo, divergencia folicular, folículo dominante, progesterona.

\section{Abstract}

The purpose of this article is to provide the reader with knowledge about the growth relationship between the future dominant follicle FD, the subordinate follicle (FS) and the intraovarian factors that influence follicular divergence in cattle.

Development. This review begins by presenting the importance of follicular divergence, when a significant difference is observed in the growth rate between the first two follicles. Thereafter, the aspects that influence this divergence are covered: presence of the corpus luteum (CL), its relationship with the dominant follicle (DF) and plasma progesterone concentrations (P4), in addition to the endocrine and molecular factors related to the follicular divergence. It is concluded that as more in-depth research is carried out, new factors that influence this process are discovered.

Keywords: Corpus luteum, follicular divergence, dominant follicle, progesterone.

\section{Resumo}

O propósito deste estudo é promover para o leitor conhecimento sobre a relação do crescimento do futuro folículo dominante FD, folículo subordinado FS e os fatores intra ovarianos que incidem na divergência folicular dos bovinos.

Metodologia: Esta revisão apresenta a importância da divergência folicular, momento no qual se observa uma diferença significativa na taxa de crescimento entre os dois primeiros folículos. Após este processo, observa-se diferentes aspetos que influência na divergência: a presença do corpo lúteo (CL), sua relação com o folículo dominante (FD) e as concentrações plasmáticas de progesterona (P4), além dos fatores endócrinos e moleculares relacionados com a divergência folicular. Conclusão: é necessário realizar mais investigações para continuar descobrindo novos fatores que interagem neste processo.

Palavras chaves: Corpo luteo, divergência folicular, folículo dominante, progesterona. 
Ingris Yohana Hernández Martínez, MVZ, MSc., Jenny Johanna Pacheco López, Ing. Esp., 3 Ana Paula Castro Santos, MV. MSc, José Nelio de Souza Sales, MV, MSc, PhD

\section{Introducción}

La divergencia folicular es el inicio de la diferencia significativa en la tasa de crecimiento del futuro folículo dominante y el mayor folículo subordinado [1], asociado con eventos endocrinos y celulares [2].

Existen cuatro principales teorías que abordan el proceso de divergencia folicular: en primer lugar, según Acosta, está relacionada con la angiogénesis perifolicular y la atresia asociada a la perfusión sanguínea del folículo, confirmando que la supresión sanguínea favorece el crecimiento de determinados folículos [3]. En segundo lugar, Fortune menciona que el aumento del factor de crecimiento semejante a insulina tipo 1(IGF-1) amplifica la respuesta a la hormona folículo estimulante (FSH), que a su vez estimula el estradiol (E2). Este aumento podría ejercer retroalimentación negativa sobre la secreción de FSH y así evitar que otros folículos de la cohorte adquieran receptores de la proteína plasmática asociada al embarazo (PAPP-A) [4]. En tercer lugar, Luo expone la siguiente teoría: "El futuro folículo dominante obtiene receptores para la hormona luteinizante ( $\mathrm{LHr}$ ) en las células de la granulosa" [5]. Finalmente, Ginther manifiesta que la divergencia folicular está influenciada por la posición ipsilateral del CL y su relación con el futuro folículo dominante [6], [7] y por la concentración de P4 [8] y [9].

El número de ondas por ciclo estral está asociado con la duración del ciclo estral y la fase luteína [10]. De acuerdo con esta observación, Sartori et al., [11] expone que en animales con ciclo estral de tres ondas, este normalmente es mayor (16,3 d) que en animales que presentan dos ondas foliculares, esto como consecuencia del retraso en el tiempo de la luteólisis [11]. Ginther et al., reportaron que en un patrón de tres ondas foliculares, el tamaño del FD de la segunda onda es mayor (16,4 \pm 0,5 mm) que el FD de la primera y tercera onda (14,6 $\pm 0,5 \mathrm{~mm}$ ) [12]. Según Borges et al., una posible explicación para la diferencia en los diámetros foliculares, sería la concentración plasmática de progesterona, lo cual demuestra que la primera onda folicular coincide con la baja concentración de P4 (CL en formación), lo que expone un feedback negativo en el hipotálamo para la liberación de $\mathrm{GnRH}$. Durante el crecimiento de la segunda onda, el CL totalmente formado secreta mayor cantidad de P4, suficiente como para aumentar el feedback negativo en el hipotálamo reduciendo la liberación de LH en la hipófisis [13]. La tercera onda folicular coincide con la reducción de concentración de P4 debido a la luteolisis [10]. En igual forma, Ginther et al., confirma la hipótesis de Borges et al., al observar una mayor concentración plasmáticas de P4 y una menor concentración de LH durante la segunda onda [14]. 


\section{Crecimiento folicular y selección del futuro folículo dominante}

En 1960 iniciaron los estudios sobre la fisiología ovárica. En esa época, aún no se conocía el patrón de crecimiento folicular, pero existían dos vertientes: una que los folículos se desarrollaban en dos ondas foliculares; y la otra que el crecimiento folicular era continuo e independiente de las fases del ciclo estral [15], [16]; estas teorías fueron usadas por muchos años, a partir de los cuales se realizaron diversos estudios con resultados contradictorios acerca de la naturaleza del crecimiento folicular.

Pierson y Ginther fueron los primeros en utilizar la ultrasonografía en ovarios de bovinos, determinando la presencia de ondas foliculares (OFs) durante el ciclo estral [17]. Cada onda folicular dura aproximadamente 10 días (variación de 6 a 10 días) [1] y [18]. En las especies monovulares, los ciclos estrales también presentan dos OFs [19], cuya maduración del FD coincide con la regresión del CL y, consecuentemente, la ovulación después de la luteólisis fisiológica [20]. Estudios realizados en novillas de la raza Holstein demostraron la predominancia de dos y tres OFs [21] y [22]. Por otra parte, en animales de la raza Nelore describieron la presencia de hasta cuatro OFs por ciclo estral [23] y [24].

Cada onda de crecimiento folicular en los bovinos involucra tres fases: reclutamiento, selección y dominancia. En el inicio de la onda, un grupo de folículos es reclutado, dando inicio a la fase de reclutamiento, en la que se exhibe crecimiento simultáneo por tres días [25]. La fase de selección folicular ocurre cuando el FD crece continuamente y los demás folículos o subordinados reducen la tasa de crecimiento y entran en atresia. Esa mudanza en la tasa de crecimiento es conocida como divergencia o desvió folicular, estableciéndose así la tercera fase, conocida como dominancia folicular [20] y [27].

El término divergencia (o desvío folicular) es definido como el momento en el cual existe una diferencia significativa en las tasas de crecimiento entre los dos folículos mayores, siendo marcada por la continuidad en el desarrollo del futuro FD y la declinación de crecimiento del segundo folículo más grande [22].

Después de la divergencia, el FD se convierte en anovulatorio debido a la presencia de altos niveles de P4, promoviendo la reducción de la frecuencia en la pulsatilidad de LH. A partir de ese momento, comienza el proceso de atresia y pérdida de la dominancia, dando inicio a una nueva onda folicular [22].

Después de una evaluación retrospectiva de los folículos a lo largo de la dinámica folicular, se puede observar que la primera diferencia de diámetro ocurre en promedio de 3 a 4 días a partir del inicio de la onda. Desde la emergencia, el futuro FD 
Ingris Yohana Hernández Martínez, MVZ, MSc., Jenny Johanna Pacheco López, Ing. Esp., 5 Ana Paula Castro Santos, MV. MSc, José Nelio de Souza Sales, MV, MSc, PhD

posee un mayor diámetro folicular $(4,0 \mathrm{~mm})$, ventaja que se mantiene hasta el momento de la divergencia, que ocurre alrededor de 2,8 días después de la emergencia de la onda. Durante esa fase la tasa de crecimiento es similar entre los dos folículos más grandes; sin embargo, una pequeña diferencia en el diámetro entre ambos sería suficiente para establecer la dominancia por el mayor folículo, antes de que el segundo mayor folículo pueda alcanzar el diámetro similar [1] y [22]. De esta forma, se considera que existe una ventaja significativa del diámetro del futuro FD, que emerge de 6 a 7 horas antes del mayor FS en el momento de la emergencia folicular (4,2 \pm 0,8 $\mathrm{mm}$ vs. 3,6 $\pm 0,1 \mathrm{~mm})$ [28].

El mecanismo de desvío es activado rápidamente cuando el FD alcanza el diámetro (8,5 mm, Bos taurus) suficiente para bloquear la secreción de FSH en la hipófisis. El rápido establecimiento del mecanismo de desvío bloquea el crecimiento del FS antes de que el mismo alcance el diámetro similar al FD [25].

En novillas de la raza Holstein (Bos taurus), la desviación folicular inicia alrededor de 2,8 días después de la ovulación, cuando el futuro FD alcanza 8,5 mm y el segundo folículo más grande alcanza 7,2 mm [22] y [25].

En relación con la raza Bos indicus, el momento de la divergencia folicular presenta variación entre los estudios. Según Castilho, la desviación folicular ocurre 3,5 días después de la ovulación, cuando el mayor folículo alcanza 7,2 mm y el segundo mayor 5,5 mm [29]. En otro estudio se verificó que en novillas de la raza Nelore (Bos indicus) la divergencia folicular inicia 2,5 días después de la ovulación, cuando los diámetros del FD y FS alcanzan 5,7 mm y 5,5 mm, respectivamente [11] y [24]. En otras investigaciones se verificó que la desviación inició 2,6 días después de la ovulación con el FD alcanzando 7,5 mm y el FS 6,2 mm [30] y [31]. Sin embargo, Bastos demostró que, en vacas Bos indicus adultas no lactantes, la desviación inicio con un diámetro entre 6,1 y 8,2 $\mathrm{mm}$ [32].

Aunque existe una diferencia entre el FD y el FS entre Bos taurus y Bos indicus, esta no es significativa, ya que el momento de la divergencia durante la primera onda folicular en relación con la ovulación anterior en Bos indicus (2,3 - 2,8 días) [24], siendo similar a lo encontrado en Bos taurus [25].

Los animales de la especie Bos indicus presentan mayor número de folículos pequeños en los ovarios, menor tamaño máximo del FD y menor CL, en comparación con los Bos taurus [33] y [34]. Las vacas o novillas Bos indicus, aunque ovulan folículos menores y con menor $\mathrm{CL}$, presentan mayores concentraciones circulantes de estradiol (E2), insulina, P4 e IGF-1, en relación con las Bos taurus [34].

La selección del FD capaz de ovular dentro de un grupo de folículos antrales es un proceso dinámico regulado por interacciones entre gonadotropinas, factores 
de crecimiento y sustancias intraovarianas [35], lo que conlleva una fase de transición crítica para el desarrollo folicular. La divergencia folicular está asociada con eventos endocrinos y celulares, como el descenso de los niveles de FSH, aumento de las concentraciones circulantes de $17 ß$-estradiol, aumento del factor de crecimiento semejante a la insulina (IGF) y aumento en la expresión de receptor de LH en las células de la célula granulosa ( $\mathrm{LHr})$ [2].

\section{Factores endocrinos y moleculares relacionados en la divergencia folicular}

Algunos estudios sugieren la existencia de factores encontrados en el líquido folicular, especialmente los factores de crecimiento, tales como: inhibina, activina, factor de crecimiento semejante a la insulina (IGFs) y sus proteínas transportadoras (IGFBP), que desempeñan un papel importante en la regulación del crecimiento, diferenciación y función folicular [36]. La variación entre ellos determina cuál es el futuro FD en cada onda. El momento crucial para que ocurra la divergencia folicular es cuando ocurren alteraciones en respuesta a las gonadotropinas [37]. Tal proceso se da cuando el folículo alcanza 8,0 mm en Bos taurus [38] y 7,5 mm en Bos indicus [7] y [31].

El aumento de LH en el momento de la divergencia estimula los receptores de LH en las células de la teca del FD y, por medio de la cascada de segundos mensajeros, aumenta la producción de andrógenos, estimulando el factor IGF [39]. Las actividades autocrinas y paracrinas del E2 en las células de la granulosa aumentan la actividad de la enzima aromatasa, aumentando también la sensibilidad de las gonadotropinas y en efecto local la liberación de IGF-1 de las proteínas transportadoras (IGFBP) [37]. Ginther et al., [1] considera que el segundo mayor folículo no consigue resistir las bajas concentraciones de FSH, haciendo que no sea capaz de asumir las características bioquímicas semejantes al FD. Al inicio de la divergencia folicular, entre las primeras 10 a 20 horas, las concentraciones de FSH disminuyen debido al incremento de la producción de E2 e inhibina por las células de la granulosa del FD, dando como resultado la pérdida de los FS [1] y [26].

Fortune relata, de otra forma, el establecimiento de la desviación. Él estima que el primer factor determinante para el desvío folicular es la inducción de una proteasa de la proteína ligado a factores de crecimiento semejantes (PPAP-A, Proteína sérica A asociada a preñez), seguida del aumento en el IGF-1 libre, que estimula la tasa de crecimiento folicular y la producción de E2 [40]. 
Ingris Yohana Hernández Martínez, MVZ, MSc., Jenny Johanna Pacheco López, Ing. Esp., 7 Ana Paula Castro Santos, MV. MSc, José Nelio de Souza Sales, MV, MSc, PhD

\section{La Hormona Folículo Estimulante (FSH)}

Está asociada con la emergencia de la onda de crecimiento folicular durante el ciclo estral [22]. En bovinos, las concentraciones plasmáticas de FSH inician 1 a 2 días antes de cada onda folicular, cuando el folículo mayor posee de 4,0 a 5,0 $\mathrm{mm}$ de diámetro [41]; y terminan aproximadamente 3 días después del inicio de la divergencia folicular, tiempo durante el cual el FD continúa desarrollándose, cambiando su dependencia de gonadotrofina primaria de FSH a LH [42]. Sin embargo, las concentraciones de FSH mantienen los niveles basales hasta que el FD pierde su dominancia, lo que genera un nuevo aumento en los niveles de FSH y emergencia de la siguiente onda [26].

Algunos trabajos realizados utilizando tratamientos con FSH al inicio del desarrollo folicular, demostraron que cualquier folículo sano o en crecimiento es capaz de tornarse dominante [26] y [43]. Con base en esta información, se desarrollaron los protocolos de superovulación con FSH para la recolección de embriones. Además, al aspirar el FD después de la desviación folicular, el FS se convierte en el dominante mediante un aumento de concentración de FSH [44]. Asimismo, un folículo elegido al azar de un grupo de folículos de $5 \mathrm{~mm}$ al inicio de la onda folicular, puede tornarse dominante después de haber sido aspirados los demás folículos [45].

Por otra parte, mediante estudios in vitro, se observó la influencia de la FSH en la producción de E2, IGF-1, activina-A e inhibina-A [25] y [46], demostrando que a mayor concentración de E2 e inhibina, la liberación de FSH por la hipófisis es menor [47].

\section{El estradiol (E2)}

Hormona producida por las células de la granulosa de folículos en crecimiento, inicia su aumento en el momento de la divergencia folicular. Esto se asocia a la elevación transitoria de LH que acompaña la selección folicular [9] y [25]. Una característica bien definida del FD es la mayor capacidad de producir E2 que los folículos reclutados o en regresión [40]. En novillas de raza Holstein, el primer aumento en la diferencia de concentración de E2, entre el primero y el segundo mayor folículo, ocurre entre 7,6 y $7,7 \mathrm{~mm}[25]$.

Algunos estudios verifican que el cambio de concentración de E2, en el momento de la desviación, están asociados con la concentración de FSH circulante, indicando la relación funcional entre las dos hormonas [41] y [48]. Por tanto, el aumento de E2 plasmático producido por el FD, en el momento de la divergencia esperada, está relacionado con la supresión de las concentraciones de FSH [41]. 
8 Factores endocrinos involucrados en la divergencia y la dominancia folicular en bovinos

\section{La Hormona Luteinizante (LH)}

Glicoproteína secretada por la hipófisis anterior, es reguladora primaria de la función ovariana. La LH puede presentar diversas funciones en la divergencia folicular, sobre todo durante la fase final del crecimiento [49]. La elevación transitoria en las concentraciones de LH inicia 32 horas antes de la divergencia, periodo en el cual el folículo pasa a responder de LH a FSH [28], hasta un periodo de 48 horas después de esta [1].

El momento de adquisición de los receptores de LHr por las células de la granulosa y su importancia en la dominancia y desvió folicular, todavía no están completamente claros. Algunas evidencias de adquisición de LH fueron encontradas en el momento de la divergencia folicular (7,0 - 9,0 mm en novillas Bos taurus). Dentro de estas están la adquisición de receptores de LH y la dependencia de LH para el crecimiento [25]. No obstante, en algunos trabajos se obtuvo el mismo resultado en diferentes diámetros foliculares [4] y [50]. En un experimento realizado en novillas Bos taurus, fueron observadas diferencias en la expresión de RNAm para LHr entre los dos mayores folículos, cuando el FD presentó entre 8,0 y 8,4 mm [51]. Estos resultados se corroboran con estudios anteriores que demuestran adhesión de LH a las células de la granulosa solamente en casos donde hay folículos con dominancia establecida [42] y [52].

En vacas Nelore, la expresión de RNAm de LHr en las células de la granulosa tiene relación con el diámetro folicular después de $7 \mathrm{~mm}$ [7], incrementando su expresión a partir de $8 \mathrm{~mm}$ [53]. Ereno et al., en su estudio realizado con novillas Nelore, observaron que las LHr funcionales en las células de la granulosa comienzan a aumentar en el momento de la divergencia folicular, siendo evidente en los folículos dominantes en comparación con los subordinados [52].

\section{Inhibina y activina}

Son fracciones proteicas del líquido folicular capaces de producir modificaciones en las concentraciones de la FSH circulante [43]. Las inhibinas son glicoproteínas dimétricas que poseen dos subunidades a y $\beta$. Esas hormonas son producidas por las células de la granulosa de folículos en crecimiento [54]. La asociación de inhibina y E2 inhibe la liberación de FSH por la hipófisis anterior [22].

No obstante, la inhibina-A producida por los folículos pequeños en crecimiento parece ser el supresor más importante de FSH durante los dos primeros días de la onda emergente; a partir de ese punto, el E2 es sintetizado por el folículo dominante y pasa a ser el principal supresor de FSH [41]. 
Ingris Yohana Hernández Martínez, MVZ, MSc., Jenny Johanna Pacheco López, Ing. Esp., 9 Ana Paula Castro Santos, MV. MSc, José Nelio de Souza Sales, MV, MSc, PhD

En un estudio realizado en novillas, no se observaron diferencias en las concentraciones de folistatina e inhibina-A del líquido folicular, en los tres folículos más grandes [54] y [35]. Del mismo modo, Beg et al., [51] utilizando como momento de referencia la divergencia folicular $(8,5 \mathrm{~mm})$, no encontraron diferencia en la cantidad total de inhibina e inhibina-A entre el folículo dominante y el mayor subordinado. Por otro lado, Austin et al., [35] observaron un aumento significativo de inhibina (>48 kDA) en el folículo dominante, solo al inicio de la inda (5 a 33 horas después del pico de FSH), manteniéndose igual en los cuatro mayores folículos después de la divergencia folicular y la disminución de FSH [35].

La activina es un homodímero de la subunidad $\beta$ que tiene acciones opuestas a la inhibina tanto en la hipófisis como en los ovarios. La activina aumenta las adquisiciones de receptores de FSH y la síntesis de inhibina por las células de la granulosa, promoviendo la proliferación de las células de la granulosa y los esteroides durante el crecimiento folicular precoz. La interacción activina-receptor es regulada por la folistatina, su proteína transportadora. La folistatina neutraliza la función de la activina en la hipófisis y en los ovarios ya que posee actividad semejante a la inhibina [54].

\section{Factor de crecimiento semejante a la insulina (IGF)}

El sistema IGF posee diversos componentes, que incluyen (IGF-1 e IGF-2) y sus diferentes proteínas y proteasas (IGFBP-1, 2, 3, 4, 5 y 6) [40], teniendo esta capacidad de actuar por vía endocrina, así como por mecanismo autocrino o paracrino [55]. La IGF-1 es un péptido de bajo peso molecular que estimula la proliferación de las células foliculares en estados iniciales y la producción de andrógenos por las células de la teca [56].

En un estudio in vitro, el IGF-1 estimuló la producción de E2 y aumentó el diámetro folicular, demostrando su relación con el crecimiento de los dos mayores folículos [57]. En otro estudio in vitro, al inyectar IGF-1 en el folículo subordinado de mayor tamaño en el momento de la desviación, se observó un aumento de E2, lo que produjo un incremento del diámetro y del flujo sanguíneo de su pared, ejerciendo influencia en su dominancia [58].

La disponibilidad de IGF libre en el folículo está determinada por las IGFBPs que modulan el crecimiento folicular [40]. La mayoría de los trabajos indican que las proteínas de IGF (IGFBPS) inhiben las acciones de las gonadotropinas en el crecimiento y diferenciación folicular [59]. 


\section{Prostaglandina}

Es secretada por el endometrio en acción luteolítica, provocando luteolisis, lo cual determina la disminución de las concentraciones de P4 [60] y [62]. Esta es utilizada en bovinos para sincronizar la ovulación [33].

En un estudio con novillas Bos taurus, se observó que la inducción de la luteolisis con PGF2a cuando el FD y el CL estaban en posición ipsilateral, lo que produjo alteración en el perfil de crecimiento folicular y cambios en la condición de dominancia para el subordinado, en vacas de diámetro folicular de 8,5 mm. Tal cambio está asociado con la presencia de CL ipsilateral al FD [6] y [61]. Estos hallazgos confirman la hipótesis del trabajo realizado por Siddinqui, en el cual se afirma que el FS se mantiene viable luego de haber ocurrido la divergencia folicular, logrando inclusive convertirse en FD [62].

\section{Conclusiones}

La relación entre las diferentes hormonas y sus receptores en la dominancia folicular, constituye un factor importante en el momento de la divergencia folicular. A medida que se realizan investigaciones más profundas sobre la dominancia folicular, se descubre que cada hormona, proteína y molécula, al igual que los sistemas reguladores de este proceso, pueden hacer que el FD pierda dominancia y que el FS pueda llegar a ocupar su posición. Por esto, es necesario que cada día el conocimiento que se tenga acerca de estos procesos sea documentado y compartido para llegar a afianzar la certeza de sus efectos y así poder integrarlo a las áreas de biología molecular.

\section{Referencias}

[1] Ginther OJ, Beg MA, Bergfelt DR, Donadeu FX, Kot K. Selection of in Monovular Species. Biol. Reprod. 2001; 65 (3): p. 638-647.

[2] Sartori R, Fricke PM, Ferreira JCP, Ginther OJ, Wiltbank MC. Follicular Deviation and Acquisition of Ovulatory Capacity in Bovine Follicles1. Biol. Reprod. 2001; 65 (5): p. 1403-1409.

[3] Acosta TJ, Hayashi KG, Matsui M, Miyamoto A. Changes in FollicularVascularity during the First Follicular Wave in Lactating Cows. J. Reprod. Dev. 2005; 51 (2): p. 273-280. 
Ingris Yohana Hernández Martínez, MVZ, MSc., Jenny Johanna Pacheco López, Ing. Esp., Ana Paula Castro Santos, MV. MSc, José Nelio de Souza Sales, MV, MSc, PhD

[4] Fortune JE, Rivera GM, Yang MY. Follicular development: The role of the follicular microenvironment in selection of the dominant follicle. Anim. Reprod. Sci. 2004; 82-83, p. 109-126.

[5] Luo W, Gumen A, Haughian JM, Wiltbank MC. The Role of Luteinizing Hormone in Regulating Gene Expression During Selection of a Dominant Follicle in Cattle. Biol. Reprod. 2011; 84 (2), p. 369-378.

[6] Ginther OJ, Siddiqui MAR, Baldrighi JM, Hoffman MM. Intraovarian factors associated with switching of a future dominant follicle to a subordinate follicle during induced luteolysis in heifers. Theriogenology. 2015; 83 (5): p. 786-796.

[7] Martinez IHY, et al. Molecular and endocrine factors involved in future dominant follicle dynamics during the induction of luteolysis in Bos indicus cows. Theriogenology. 2018; 111: p. 78-83.

[8] Ginther OJ, Dangudubiyyam SV. Role of intraovarian mechanisms and side of ovary on characteristics of follicle selection in Bos taurus heifers. Theriogenology. 2019; 135: p. 56-64.

[9] Ginther OJ, Bergfelt DR, Beg MA, Kot K. Follicle Selection in Cattle: Role of Luteinizing Hormone1. Biol. Reprod. 2001; 64 (1): p. 197-205.

[10] Borges AM, et al. Dinâmica folicular e momento da ovulação em vacas não lactantes das raças Gir e Nelore durante duas estações do ano. Arq. Bras. Med. Vet. e Zootec. 2004; 56 (3): p. 346-354.

[11] Sartori R, Haughian JM, Shaver RD, Rosa GJM, Wiltbank MC. Comparison of ovarian function and circulating steroids in estrous cycles of Holstein heifers and lactating cows. J. Dairy Sci. 2004; 87 (4): p. 905-920.

[12] Ginther OJ, Knopf L, Kastelic JP. Temporal associations among ovarian events in cattle during oestrous cycles with two and three follicular waves. J. Reprod. Fertil. 1989; 87 (1): p. 223-230.

[13] Neto JR, da Costa EP, de Moraes Ferreira A, de Sá WF, dos Santos GM, Arroyo RJO. Utilização de estrógeno exógeno no início do ciclo estral em vacas leiteiras mestiças. Rev. Bras. Zootec. 2011; 40 (7): p. 1504-1511.

[14] Ginther OJ, Domingues RR, Dangudubiyyam SV, Araujo ER. Gonadotropin concentrations associated with variations in diameter deviation during follicle selection in Holstein heifers. Anim. Reprod. Sci. 2018; 192: p. 271-279. 
[15] Rajakoski E. The ovarian follicular system in sexually mature heifers with special reference to seasonal, cyclical, and left-right variations. Obstet. Gynecol. Surv. 1960; 34 (Suppl 52): p. 836-838, 1960.

[16] Senger PL. Pathways to pregnancy and parturition. Segunda edición. Ciudad: Pullman WA. Casa editorial: Current Conceptions, Inc.; 2003. p. 1-381.

[17] Pierson RA, Ginther OJ. Ultrasonography of the bovine ovary. Theriogenology. 1984; 21 (3): pp. 495-504.

[18] Lucy MC, Savio JD, Badinga RL, De la Sota RL, Thatcher WW. Factors that affect ovarian follicular dynamics in cattle 1. J. Anim. Sci. 1992; 70 (11): p. 3615-3626.

[19] Ginther OJ, Bergfelt DR, Beg MA, Kot K. Follicle Selection in Cattle: Relationships among Growth Rate, Diameter Ranking, and Capacity for Dominance 1. Biol. Reprod. 2001; 65 (2): p. 345-350.

[20] Sirois J, Fortune E. Ovarian Follicular Dynamics during the Estrous Cycle Ultrasonography' in Heifers Monitored by. Biol. Reprod. 1988; 39: p. 308-317.

[21] Ginther OJ, Bergfelt DR, Kulick LJ, Kot K. Selection of the Dominant Follicle in Cattle. Biol. Reprod. 2000; 63 (2): p. 383-389.

[22] Ginther OJ, Kot K, Kulick LJ, Wiltbank MC. Emergence and deviation of follicles during the development of follicular waves in cattle. Theriogenology. 1997; 48 (1), p. 75-87.

[23] Figueiredo RA, Barros CM, Pinheiro OL, Soler JMP. Ovarian follicular dynamics in nelore breed (Bos indicus) cattle. Theriogenology. 1997; 47: pp. 1489-1505.

[24] Sartorelli ES, Carvalho LM, Bergfelt DR, Ginther OJ, Barros CM. Morphological characterization of follicle deviation in Nelore (Bos indicus) heifers and cows. Theriogenology. 2005; 63: p. 2382-2394.

[25] Ginther OJ, Beg MA, Kot K, Meira C, Bergfelt DR. Associated and Independent Comparisons Between the Two Largest Follicles Preceding Follicle Deviation in Cattle 1. Biol. Reprod. 2003; 68 (2): p. 524-529.

[26] Bodensteiner KJ, Wiltbank MC, Bergfelt DR, Ginther OJ. Alterations in follicular estradiol and gonadotropin receptors during development of bovine antral follicles. Theriogenology. 1996; 45 (2): p. 499-512. 
Ingris Yohana Hernández Martínez, MVZ, MSc., Jenny Johanna Pacheco López, Ing. Esp., 13 Ana Paula Castro Santos, MV. MSc, José Nelio de Souza Sales, MV, MSc, PhD

[27] Fortune JE. Antral follicles: Recruitment and selection of ovulatory follicles. Segunda edición. Elsevier. 2018; 2: p. 80-86.

[28] Kulick LJ. Kot K, Wiltbank MC, Ginther OJ. Follicular and hormonal dynamics during the first follicular wave in heifers. Theriogenology. 1999; 52 (5): p. 913-921.

[29] Castilho C. Divergência no crescimento folicular em novilhas da raça nelore e sua influência sobre a competência oocitária para o desenvolvimento embrionário in vitro. Tesis de doctorado Universidade Estadual Paulista. Ciudad Jaboticabal. 2003; p. 1-86

[30] Castilho C, Garcia JM, Renesto A, Nogueira GP, Brito LFC. Follicular dynamics and plasma FSH and progesterone concentrations during follicular deviation in the first post-ovulatory wave in Nelore (Bos indicus) heifers. Anim. Reprod. Sci. 2007; vol. 98 (3-4): p. 189-196.

[31] Gimenes LU, et al. Follicle deviation and ovulatory capacity in Bos indicus heifers. Theriogenology. 2008; 69 (7), p. 852-858.

[32] Bastos M. Diferenças fisiológicas reprodutivas entre Bos taurus e Bos indicus. in Aleph, 2012. Tesis de doctorado. Universidade Estadual Paulista. Facultad de medicina Veterinaria y Zootecnia. Botucatu. p.1-108.

[33] Bó GA, Baruselli PS, Martínez MF. Pattern and manipulation of follicular development in Bos indicus cattle. Anim. Reprod. Sci. 2003; 78: p. 307-326.

[34] Sartori R, Bastos M, Baruselli P, Gimenes L, Ereno R, Barros C. Physiological differences and implications to reproductive management of Bos taurus and Bos indicus cattle in a tropical environment. Soc. Reprod. Fertil. Suppl. 2020; 67: p. 357-375.

[35] Austin EJ, et al. Alterations in Intrafollicular Regulatory Factors and Apoptosis During Selection of Follicles in the First Follicular Wave of the Bovine Estrous Cycle 1. Biol. Reprod. 2001; 64 (3): p. 839-848.

[36] Ireland JJ, Roche JF. Development of antral follicles in cattle after prostaglandin-induced luteolysis: Changes in serum hormones, steroids in follicular fluid, and gonadotropin receptors. Endocrinology. 1982; 111, pp. 2077-2086.

[37] Ginther OJ, Gastal EL, Gastal MO, Checura CM, Beg MA. Dose-Response Study of Intrafollicular Injection of Insulin-Like Growth Factor-I on Follicular Fluid Factors and Follicle Dominance in Mares1. Biol. Reprod. 2004; 70 (4): p. 1063-1069. 
[38] Ginther OJ, Bergfelt DR, Kulick LJ, Kot K. Selection of the dominant follicle in cattle: Establishment of follicle deviation in less than 8 hours through depression of FSH concentrations. Theriogenology. 1999; 52 (6): p. 1079-1093.

[39] Vásquez-Cano JF, Olivera M. Señalización celular en el folículo antral bovino. Orinoquia. 2010; 14 (2): 178-187.

[40] Fortune JE, Rivera GM, Evans AC, Turzillo AM. Differentiation of Dominant Versus Subordinate Follicles in Cattle1. Biol. Reprod. 2001; 65 (3): p. 648-654.

[41] Ginther OJ, Bergfelt DR, Kulick LJ, Kot K. Selection of the Dominant Follicle in Cattle: Role of Estradiol1. Biol. Reprod. 2000; 63 (2): p. 383-389.

[42] Webb R, Campbell B, Garverick H, Gong J, Gutiérrez C, Armstrong D. Molecular mechanisms regulating follicular recruitment and selection. Biosci. Proc. 1999; 66: p. 33-48.

[43] Adams GP, Kot K, Smith CA, Ginther OJ. Selection of a dominant follicle and suppression of follicular growth in heifers. Anim. Reprod. Sci. 1993; 30 (4): p. 259-271.

[44] Ginther OJ, Bergfelt DR, Kulick LJ, Kot K. Selection of the Dominant Follicle in Cattle: Role of Two-Way Functional Coupling between Follicle-Stimulating Hormone and the Follicles1. Biol. Reprod. 2000; 62 (4): p. 920-927.

[45] Gibbons JR, Wiltbank MC, Ginther OJ. Functional interrelationships between follicles greater than $4 \mathrm{~mm}$ and the follicle-stimulating hormone surge in heifers. Biol. Reprod. 1997; 57 (5): p. 1066-1073.

[46] Sarwar Z, et al. Meta-analysis to determine effects of treatment with FSH when there is progestin-priming on in-vitro embryo production using ovum pick-up in Bos taurus cows. Anim. Reprod. Sci. 2020; 221: p. 106590.

[47] Cheong, SH, Fortune JE, Allen JJ, Butler WR, Gilbert RO. Androgen production in response to LH is impaired in theca cells from nonovulatory dominant follicles in early-postpartum dairy cows. Domest. Anim. Endocrinol. 2020; 71: p. 106385.

[48] Chasombat J, Nagai T, Parnpai R, Vongpralub T. Ovarian follicular dynamics and hormones throughout the estrous cycle in Thai native (Bos indicus) heifers. Anim. Sci. J. 2014; 85 (1): p. $15-24$. 
Ingris Yohana Hernández Martínez, MVZ, MSc., Jenny Johanna Pacheco López, Ing. Esp., 15 Ana Paula Castro Santos, MV. MSc, José Nelio de Souza Sales, MV, MSc, PhD

[49] Driancourt MA. Regulation of ovarian follicular dynamics in farm animals. Implications for manipulation of reproduction. Theriogenology. 2001; 55 (6): p. 1211-1239.

[50] Robert C, Gagné D, Lussier JG, Bousquet D, Barnes FL, Sirard MA. Presence of LH receptor mRNA in granulosa cells as a potential marker of oocyte developmental competence and characterization of the bovine splicing isoforms. Reproduction. 2003; 125 (3): p. 437-446.

[51] Beg MA, Bergfelt DR, Kot K, Wiltbank MC, Ginther OJ. Follicular-Fluid Factors and GranulosaCell Gene Expression Associated with Follicle Deviation in Cattle1. Biol. Reprod. 2001; 64 (2): p. 432-441.

[52] Ereno RL, et al. Expression of mRNA Encoding the LH Receptor (LHR) and LHR Binding Protein in Granulosa Cells from Nelore (Bos indicus) Heifers Around Follicle Deviation. Reprod. Domest. Anim. 2015; 50 (6), p. 952-957.

[53] Simões RA, et al. Ovulation rate and its relationship with follicle diameter and gene expression of the LH receptor (LHR) in Nelore cows. Theriogenology. 2012; 77 (1), p. 139-147.

[54] Mihm M, Austin EJ. The final stages of dominant follicle selection in cattle. Domest. Anim. Endocrinol. 2002; 23 (1-2): p. 155-166.

[55] Spicer LJ. Proteolytic Degradation of Insulin-Like Growth Factor Binding Proteins by Ovarian Follicles: A Control Mechanism for Selection of Dominant Follicles1. Biol. Reprod. 2004; 70 (5): p. 1223-1230.

[56] Spicer LJ, Alonso J, Chamberlain CS. Effects of thyroid hormones on bovine granulosa and thecal cell function in vitro: Dependence on insulin and gonadotropins. J. Dairy Sci. 2001; 84 (5): p. 1069-1076.

[57] Dentis JL, Schreiber NB, Gilliam JN, Schutz LF, Spicer LJ. Changes in brain ribonuclease (BRB) messenger RNA in granulosa cells (GCs) of dominant vs subordinate ovarian follicles of cattle and the regulation of BRB gene expression in bovine GCs. Domest. Anim. Endocrinol. 2016; 55: p. 32-40.

[58] Shahiduzzaman AK, Beg MA, Palhao MP, Siddiqui MA, Shamsuddin M, Ginther OJ. Stimulation of the largest subordinate follicle by intrafollicular treatment with insulin-like growth factor 1 is associated with inhibition of the dominant follicle in heifers. Theriogenology. 2010. 74 (2): p. 194-201. 
16 Factores endocrinos involucrados en la divergencia y la dominancia folicular en bovinos

[59] Lenz-Souza MI, Ramírez GF, Uribe LF. Papel del factor de crecimiento semejante a la insulina (IGF-1_ en la regulación de la función ovárica. Biosalud. 2007; 6: p. 149-159.

[60] Shirasuna K. Nitric oxide and luteal blood flow in the luteolytic cascade in the cow. J. Reprod. Dev. 2010; 56 (1): p. 9-14.

[61] Ginther OJ, Gomez-León VE. Intraovarianism and differences in ovulation frequency between left and right ovaries in Bos taurus heifers. Reprod. Biol. 2020; 20 (3): pp. 0-1.

[62] Siddiqui MA, Ginther OJ. Switching of largest follicle from dominant to subordinate status when follicle and CL are in same ovary in heifers. Theriogenology. 2014; 82 (2): p. 259-265. 